\title{
Review of the Nutrition Guidance Irish Healthcare and Charitable Organisations and Cancer Centres provide for Cancer Patients and Cancer Survivors.
}

\section{Abstract}

Background: A cancer diagnosis presents a potential teachable moment however, evidence-based information, guidance and support all need to be available in order to capitalise on this. Health care professionals report providing little advice in this area. Previous work has shown that cancer survivors tend to prefer statutory and recognised charitable centres and groups. It is therefore important to determine what nutrition information is available on these websites as otherwise this group may turn to less reliable sources of information

Objective: To determine the availability, quality and readability of nutrition information for cancer patients and survivors from Irish healthcare organisations, cancer charity and support groups

Methods: Organisations were located using three search strategies: 1) We first sought to identify any nutrition information for cancer patients or survivors provided by the Irish Health Service Executive (HSE), 2) To identify suitable organisations the word "cancer" was searched for in the search engine of the Benefacts (official register of charitable organisations) website. The ten largest organisations were included and 3) an additional internet search was conducted using the terms 'cancer charities Ireland' and 'cancer support groups Ireland'. The first two pages of results were assessed. The quality of the nutrition information was determined using the adapted IPDAS tool. Readability testing was carried out on the websites where nutrition information was available using

Results: A total of 32 websites were assessed. Of these only five provided any form of nutrition guidance for cancer patients with three of these five also providing some guidance for cancer survivors. The guidance provided centred on guidelines and lacked practical strategies for their implementation. The quality of the information provided ranged from 19.5-29 out of a maximum of 40. The readability level required to understand the information ranged from 11-17 years of age.

Conclusion: There is a need for nutritional guidance to be provided by national health and cancer related organisations and for this information to be specific to the cohort e.g. patient vs survivor. There is also a need for practical strategies to make the translation of guidelines into practice easy for patients and survivors.

\section{Conflict of Interest}

There is no conflict of interest 